\title{
Cycloid Approximation with Circle for the Calculation of Exit Angle
}

\author{
György PÓKA, István NÉMETH
}

\begin{abstract}
Manufacturers' goal is to control, avoid or minimise burr during manufacturing. Burr control is used frequently, because it costs less and/or is less time-consuming Based on the experimental results and simulations in the case of face milling it has been stated that exit order and planar exit angle have a significant influence on burr formation. A carefully planned tool path can provide constant exit angle. Setting the exit angle to an appropriate value or range, burr can be avoided or minimised. The exit angle can be determined approximately in a simple way, using a circle as a path of tool tip. The precise value of the error of the approximated calculation of exit angle has not been revealed yet. In this paper, this error is examined. The slope of a circle and the theoretically accurate cycloid curve can be expressed analytically. The difference between the two slopes can be calculated. Its maxima or minima give the value of the error which can be deducted into simple equations that are valid either for linear or circular feed. If the exit angle is changing, the chip thickness is also changing. With the help of the resulted expressions, a special workpiece contour can be designed, where the exit angle is constant and only the chip thickness is changing. In this way, the examination of the effect of the chip thickness on burr formation will be possible.
\end{abstract}

Keywords: approximation techniques; burr formation; exit angle; face milling

\section{INTRODUCTION}

Researchers' aim is on the one hand to discover the main reasons of burr formation and on the other hand to control them. There are two different ways to deal with burr: deburring and control Numerous factors have been examined from cutting conditions, materials to tool geometry and path. Nowadays, analytical and finite element models are available [2, 3] and with the help of them burr formation can be simulated in the most often used applied machining operations, such as milling, drilling and turning. Due to the simulations, researchers found that by providing suitable circumstances, burr formation can be controlled.

Formerly, Gillespie and Blotter [7] observed that burr cannot be prevented by changing the feed, speed, or tool geometry alone. Burr formation is most significantly impacted by the material of the workpiece, tool wear, the tool path and the cutting conditions. Although changes in these parameters have a major effect on burr formation, it is still necessary to examine the entire cutting process. Burr formation at the edges can also react to the cutting process, e.g. vibrations and surface's quality $[22,28]$.

With the help of experiments, Wang and Zhang [27] confirmed that, in the case of face milling, the workpiece material and geometry, tool geometry and cutting conditions exert major effects on burr formation.

Concerning micro-slot milling, Kiswanto et al. [11] identified that tool wear, as a result of machining time, is the most influential factor of burr formation.

Kou et al. [12] used support material to avoid burr in case of micro milling. Through their experiments they showed that burrs did not appear on the workpiece surface after the instant adhesive was removed and cared about uncontaminated surface.

$\mathrm{Wu}$ et al. [29] examined the influences of the uncut chip thickness and the cutting edge radius on burr formation. It has been stated, that top burr decreased to the minimum value when the feed ratio was almost equal to the cutting edge radius. Larger burr appeared on the down milling side.

Amrendra et al. [1] investigated the finite element modelling of burr formation in high speed micromilling of
Ti6Al4V. They observed that the side exit burr at the up milling side was the biggest and the cutting speed was the major factor to reduce the burr size in micromilling.

Niknam et al. $[18,19]$ proposed an analytical model for calculating the thickness of exit burr in milling of ductile materials and found good correlations between the experimental and modelled values. It was stated that exit up milling side burr thickness could be controlled by cutting parameters, e.g., feed per tooth, depth of cut and tool geometry, but the effect of the cutting speed was negligible.

Lekkala et al. [14] also made a theoretical model of burr formation in micro-end milling. It was demonstrated that the tool diameter, depth of cut, number of flutes, feed rate and the interaction between the feed rate and number of flutes had significant effect on burr formation. Their study shows that there are six different types of burr formation mechanisms in micro-end milling of aluminium and stainless steel.

Olvera and Barrow [20] examined the effect of coated and uncoated inserts and found that there is only a small difference in burr size. The comparison of inserts with nose radiuses and with wiper blades resulted in the latter one producing smaller burr. They observed that, by switching from up-milling to down-milling, the size of burr can be reduced efficiently, and in some cases it can even be eliminated.

Jones and Furness [10] examined the face milling of aluminium. They found that burr size is minimal when the exit angle is between $76^{\circ}$ and $118^{\circ}$, regardless of tool wear. In addition, high feed, low speed, new tools and harder materials more effectively contributed to the reduction of burr formation.

Chern [4] tested different aluminium alloys and realised that the most efficient way to reduce burr was the change of the planar exit angle. Chern proposed that the value of the exit angle should be about $150^{\circ}$.

Silva et al. [24,25] in their experiments found that while the usage of cutting fluid did not have a significant effect on burr formation, the tool geometry and radial depth of cut with the related exit angle did have one.

Hashimura et al. [8] described and classified burrs in case of face milling. They observed that the size of burr 
was dependent on the sequence of the exit points of the tool edge (Fig. 1). In the case of Fig. 1a) the sequence is ABC, and here the size of burr will be the smallest. In the case of Fig. 1b), the sequence is CBA, and here the size of burr will be the largest.

Furthermore, Kumar [13] also researched the sequence of exit order. He found that the burr size depended on the material of the workpiece, and he concluded that the trend of the change of burr size in different cases of exit order remained the same.

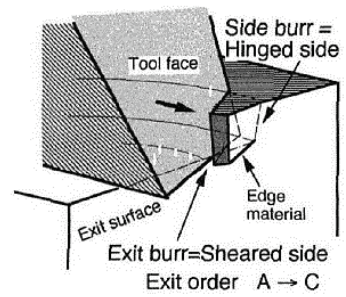

a)

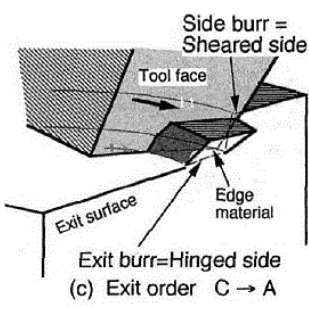

b)
Figure 1 The effect of exit order sequence on burr formation ( $A$ : minor cutting edge, $\mathrm{C}$ : major cutting edge, $\mathrm{B}$ : section of $\mathrm{A}$ and $\mathrm{B}$ ) [8]

The exit angle can be calculated if the path of tool tip and the workpiece contour are known. The theoretical path is generally substituted with simple curves. For the determination of the exit angle, Chern [5] approximated the path of a point on the cutting edge using a circle. In this case, the exit angle can be calculated using Eq. (1) in the case of linear contours (Fig. 2):

$\alpha_{t}=\pi / 2+\arcsin (e / r)$

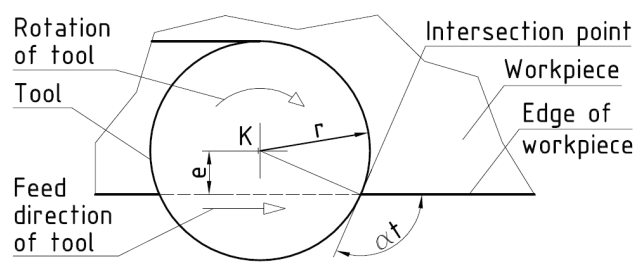

Figure 2 The exit angle in the case of face milling

With the help of this simple expression, Eq. (1), researchers examined the generation of optimised tool path with respect to burr formation.

Narayanaswami and Dornfeld [17] divided a workpiece contour consisting of lines into primary and secondary burr zones. In the scope of their research, burr formation was estimated based on whether the exit angle was greater than a predefined value. In this case, the exit angle was calculated with the help of the tool geometry, the location of the centrepoint of the tool and the workpiece contour.

Hassamontr et al. [9] proposed a similar method to avoid burr formation. They extended the applicability of the algorithm developed by Chu and Dornfeld [6], where workpiece contours can have curves and islands, and feed direction can also be changed.

The authors of this paper in one of their previous works [21] created special tool paths that provided constant exit angle. Their experimental results were promising.

Burr-minimal tool paths are longer than conventional paths, therefore, machining time increases. Rangarajan [23] proposed the determination of a feasible offset region, in which the tool could move and burr size was foreseen to be minimal. Concerning the area inside this region, paths with a minimal length or paths matching other aspects can be generated. However, exiting at more than one points can cause problems, as in this case it is not certain whether burr formation can be avoided on one or more edges.

Since the referred methods are based on approximation, it is necessary to examine the applicability in the case of different feed rate. The following sections present new mathematical expressions with the help of which the maximum of the difference between the exact and approximated exit angles can be determined in the case of linear and circular tool paths. The resulted expressions can also aid the tool path generation.

\section{LINEAR FEED}

This section presents two solutions for the description of tooltip path in the case of linear feed with general direction.

\subsection{Coordinate System Fixed to the Workpiece}

The exit angle can be determined using the workpiece contour and the path of the cutting edge. Therefore, it is necessary to know the path of a point assigned on the cutting edge. The path is determined by rotation and linear motion and is called cycloid curve. Martellotti $[15,16]$ in his early research used cycloid curves, and later the correctness of the model was verified by Spiewak [26]. It should be noted that Martellotti substituted the cycloid curve by a circle to get a simple expression for the determination of chip thickness.

In case of face milling, the exit angle can be established at the intersection point of two curves using two tangent lines. The first curve is the workpiece contour; the second one is the path of a point assigned on the cutting edge on the radius $r$ (Fig. 3). Exit generally occurs in the case of up-milling and its value is in the range of $0^{\circ}<\alpha<$ $180^{\circ}$.

In Fig. 3, the real speed vector belonging to the real path is denoted by $\bar{v}_{r}$, while the real exit angle $\alpha_{r}$ belongs to $\bar{v}_{r}$. The path of the cutting edge can be approximated using a circle: in this case the speed vector is $\bar{v}_{t}$ and the exit angle is $\alpha_{t}$. Angle $\varphi$ can be defined with the help of the two speed vectors and is equal to the difference between the real and approximated exit angles. This angular deviation is influenced by the angular position of the tool and the actual feed rate.

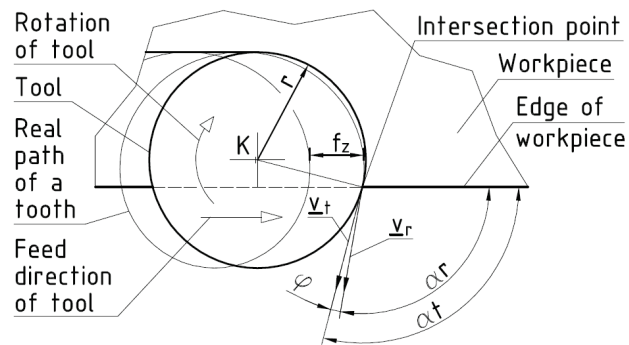

Figure 3 The difference between the real and approximated exit angles 
The applicability of the approximation using a circle can be examined by identifying the maxima and minima of $\varphi$ in the case of an arbitrary feed rate. Angle $\varphi$ can be defined as the difference between the slopes of the real and approximated curves expressed in radian.

The centrepoint of the tool $K$ moves along a line of a general position, where the position of the line is described by functions $X_{C}(t)$ and $Y_{C}(t)$ shown by Eqs. (2) and (3), respectively:

$$
\begin{aligned}
& X_{C}(t)=R \cdot\left(\left(t+t_{3}\right) \cdot \cos \left(t_{1}\right)-t_{0} \cdot \sin \left(t_{1}\right)\right) \\
& Y_{C}(t)=R \cdot\left(\left(t+t_{3}\right) \cdot \sin \left(t_{1}\right)+t_{0} \cdot \cos \left(t_{1}\right)\right)
\end{aligned}
$$

The constants belong to the locations of the line and of the centrepoint of the tool: $t_{0}$ belongs to the distance of the line measured from the origin, $t_{1}$ belongs to the angular position of the line, $t_{2}$ belongs to the tool initial angular position, and $t_{3}$ belongs to the location of the tool centrepoint on the line. In Eqs. (2) and (3), a rotation $\left(t_{1}\right)$ follows two translations $\left(R \cdot t_{3}\right.$ and $\left.R \cdot t_{0}\right)$. The tool radius is $r$, to which a concentric circle can be assigned. This circle is the rolling circle and its radius is $R$. In the case of linear feed, the rolling circle with radius $R$ rolls, without slips, on a line that is parallel to the feed direction. The ratio of the two radiuses can be expressed by $k$ according to Eq. (4):

$$
k=r / R
$$

In the case of face milling, it is generally true that $R<<r$, thus $1<<k$. Feed rate per rotation, $f$, can be expressed using the radius of the rolling circle according to Eq. (5):

$$
f=R \cdot 2 \cdot \pi=(r / k) \cdot 2 \pi
$$

The paths of tool tip draw cycloid curves. With reference to this, a general position cycloid curve with arbitrary constants $\left[t_{0}, t_{1}, t_{2}, t_{3}\right]$ can be described by functions $X(t)$ and $Y(t)$, indicated by Eqs. (6) and (7), respectively:

$$
\begin{aligned}
& X(t)=X_{C}(t)+r \cdot \cos \left(-t+t_{1}+t_{2}\right) \\
& Y(t)=Y_{C}(t)+r \cdot \sin \left(-t+t_{1}+t_{2}\right)
\end{aligned}
$$

In Eqs. (6) and (7), $t$ means the angular position of the tool and the direction of rotation is clockwise. In order that the slopes of the curves can be compared, they should be expressed in radian. Due to the use of the arcus tangent function, the slope in radian remains in the range of $-\pi / 2 \leq \alpha \leq \pi / 2$. However, if the direction of $\alpha$ is necessary for the calculation, the range should be extended to $-\pi \leq \alpha \leq \pi$. Therefore, $\alpha$ should be corrected with $\pi$, where $X^{\prime}(t)<0$, and the sign is determined by $Y^{\prime}(t)$. The correction can be expressed in a general form as follows, Eq. (8):

$$
\operatorname{corr}(t)=\pi \cdot\left(\operatorname{sgn}\left(X^{\prime}(t)\right)-1\right) /\left(2 \cdot \operatorname{sgn}\left(Y^{\prime}(t)\right)\right)
$$

Eq. (8) can be used with the following conditions: $\operatorname{sgn}\left(X^{\prime}(t)\right) \neq$ const,$\quad \operatorname{sgn}\left(Y^{\prime}(t)\right) \neq$ const,$\quad \operatorname{sgn}(0)=+1$ and $\partial \operatorname{corr}(t) / \partial t \equiv 0$. The slope of the cycloid in radian is expressed by Eq. (9) (see Fig. 4):

$$
\begin{aligned}
& \mu_{1}(k, t)=\arctan \left(Y^{\prime}(t) / X^{\prime}(t)\right)= \\
& =\arctan \left[\frac{\sin \left(t_{1}\right)-k \cdot \cos \left(-t+t_{1}+t_{2}\right)}{\cos \left(t_{1}\right)+k \cdot \sin \left(-t+t_{1}+t_{2}\right)}\right]+\operatorname{corr}(t)
\end{aligned}
$$

In Eq. (9), the radius of the tool is eliminated, thus, in this case the slope is independent of it. If $R \rightarrow 0$, then $k \rightarrow \infty$. If the feed rate is almost $0(R \rightarrow 0$ and $k \rightarrow \infty)$, Eqs. (6) and (7) describe a circle.

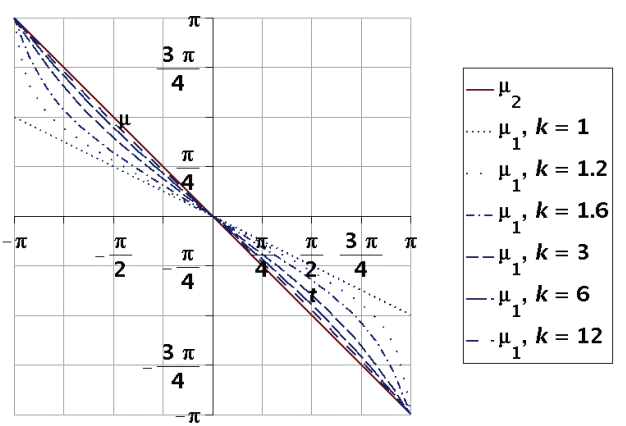

Figure 4 The slope of the circle $\mu_{1}$ and the cycloid curve $\mu_{2}$. The constants that belong to the cycloid are: $t_{0}=0, t_{1}=0, t_{2}=(\pi / 2), t_{3}=0$.

The circle and its slope in radian $\left(\mu_{2}(t)\right)$ can be expressed in a simple form. The difference between the real and approximated exit angle can be described by Eq. (10):

$$
\begin{aligned}
& \varphi(t, k)=\mu_{2}(t)-\mu_{1}(t, k)=\left(-t+t_{1}+t_{2}\right)- \\
& -\arctan \left[\frac{\sin \left(t_{1}\right)-k \cdot \cos \left(-t+t_{1}+t_{2}\right)}{\cos \left(t_{1}\right)+k \cdot \sin \left(-t+t_{1}+t_{2}\right)}\right]-\operatorname{corr}(t)
\end{aligned}
$$

To determine the maxima and minima of $\varphi(t, k)$ in the case of any $k$, the derivative function of $\varphi(t, k)$ with respect to $t$ should be used. After simplification, the equation can be formulated as Eq. (11) (see Fig. 5):

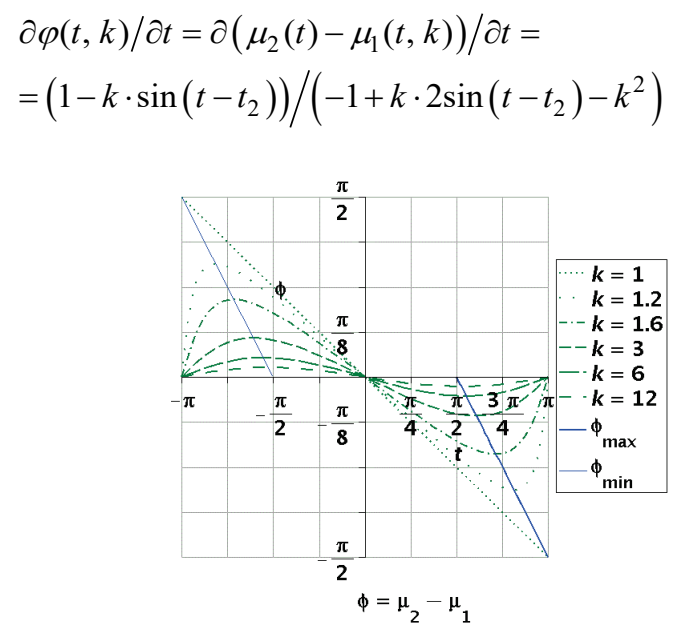

Figure 5 The difference of the slope of a circle and of a cycloid expressed in radian in the function of $k$, the constants are: $t_{0}=0, t_{1}=0, t_{2}=(\pi / 2), t_{3}=0$ 
If the denominator of Eq. (11) is equal to zero, the expression at one given point is discontinued. In order to continue with any further examination, the case when the denominator of Eq. (11) is zero has to be solved to $t$, which results in Eq. (12):

$t=t_{2}+\arcsin \left(\left(1+k^{2}\right) /(2 k)\right)$

Eq. (12) does not have a real solution if $k<1$. However, the previous assumption was $1 \leq k$, therefore, in this case singularity poses no problem. The maxima and minima of Eq. (10) can be found where the numerator of Eq. (11) is equal to zero if Eq. (11) is continuous in the examined range. By solving the numerator of Eq. (11) to $t$, the location of the maxima and minima in function of $k$ can be obtained with Eqs. (13a) and (13b), respectively:

$$
\begin{aligned}
& t=t_{2}+\arcsin (1 / k) \\
& t=t_{2}+\pi-\arcsin (1 / k)
\end{aligned}
$$

By substituting Eqs. (13a) and (13b) into Eq. (10), the maxima and minima of angular deviation $(\Phi(k))$ can be determined, respectively. Eq. (10) contains the constant $t_{1}$, but Eqs. (13a) and (13b) do not contain it, therefore, only $t_{2}$ can be eliminated. The maxima and minima of angular deviation can be expressed in simple forms with Eq. (14a) and Eq. (14b), respectively, using trigonometric identifies. The values of correction are influenced by $t_{1}$ and $k$ (see Fig. $6)$.

$$
\begin{aligned}
& \Phi(k)=\arcsin (1 / k) \\
& \Phi(k)=\pi-\arcsin (1 / k)
\end{aligned}
$$

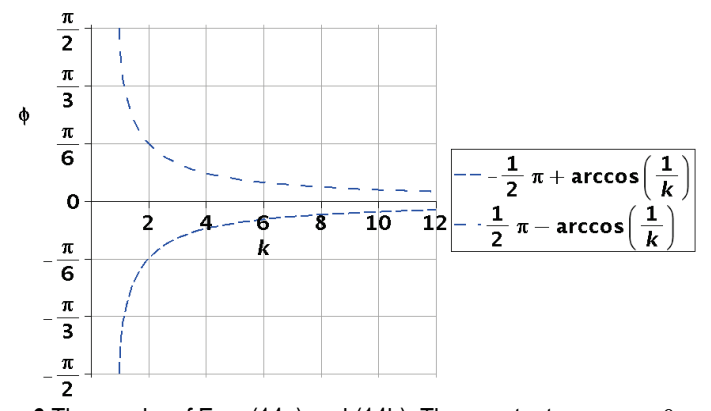

Figure 6 The graphs of Eqs. (14a) and (14b). The constants are: $t_{0}=0, t_{1}=0$, $t_{2}=(\pi / 2), t_{3}=0$.

In practice, the $\varphi(f)$ function can be applied more easily, where $f$ is the feed per rotation. Substituting Eq. (5) into Eqs. (14a) and (14b) we get Eqs. (15a) and (15b), respectively:

$$
\begin{aligned}
& \Phi(f)=\arcsin (f /(2 \cdot r \cdot \pi)) \\
& \Phi(f)=\pi-\arcsin (f /(2 \cdot r \cdot \pi))
\end{aligned}
$$

Eqs. (15a) and (15b) give the maximal or minimal values $(\Phi)$ of the angular deviation $(\varphi)$ in the function of $f$, in the case of $2 r \pi<f$. With the assumption $f<<r$, the curves of Eq. (15a) and (15b) are approximately linear, therefore, the calculation can be simplified with Eq. (16a) and Eq. (16b) (see Fig. 7), respectively:

$$
\begin{aligned}
& \Phi(f) \cong f /(r \cdot 2 \cdot \pi) \\
& \Phi(f) \cong \pi-f /(r \cdot 2 \cdot \pi)
\end{aligned}
$$

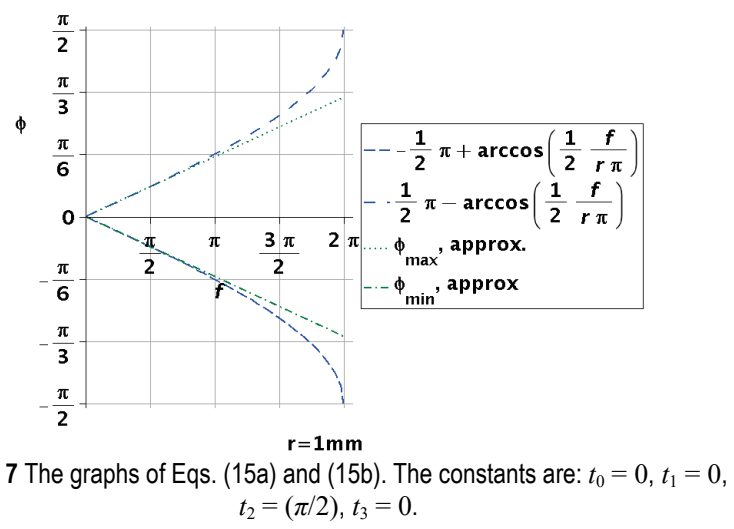

\subsection{Coordinate System Fixed to the Tool}

In the previous deduction, correction has to be used for determining the slope of the cycloid and the circle expressed in radian. However, correction is unnecessary if the cycloid curve is described in a coordinate system fixed to the tool, and if appropriate $t_{1}$ and $t_{2}$ are chosen. The condition for describing this cycloid in a coordinate system fixed to the tool is as follows: $t=\tau=t_{m}$, where $\tau$ is an arbitrary time moment, and the other constants, $\left[t_{0}, t_{1}, t_{2}, t_{3}\right]$, are arbitrary as well. Similarly to Eqs. (2) and (3), $X_{C}(t)$ and $Y_{C}(t)$ can be express as follows, Eqs. (17) and (18):

$$
\begin{aligned}
& X_{C}(t)= \\
& =R \cdot\left(\left(t-t_{m}\right) \cdot \cos \left(t_{1}-t_{m}\right)+t_{3} \cdot \cos \left(t_{1}\right)-t_{0} \cdot \sin \left(t_{1}\right)\right) \\
& Y_{C}(t)= \\
& =R \cdot\left(\left(t-t_{m}\right) \cdot \sin \left(t_{1}-t_{m}\right)+t_{3} \cdot \sin \left(t_{1}\right)-t_{0} \cdot \cos \left(t_{1}\right)\right)
\end{aligned}
$$

In Eqs. (17) and (18), the sequence of transformations is the following: translation $\left(R \cdot t_{m}\right)$, rotation $\left(t_{m}\right)$, translation $\left(R \cdot t_{3}\right.$ and $\left.R \cdot t_{0}\right)$ and rotation $\left(t_{0}\right)$. A cycloid curve in a coordinate system fixed to the tool can be described by functions $X(t)$ and $Y(t)$ as shown by Eq. (19) and Eq. (20), respectively:

$$
\begin{aligned}
& X(t)=X_{C}(t)+r \cdot \cos \left(-t+t_{m}+t_{1}+t_{2}\right) \\
& Y(t)=Y_{C}(t)+r \cdot \sin \left(-t+t_{m}+t_{1}+t_{2}\right)
\end{aligned}
$$

The slope in radian of the cycloid without correction is expressed by Eq. (21):

$$
\begin{aligned}
& \mu_{1}(k, t)=\arctan \left(Y^{\prime}(t) / X^{\prime}(t)\right)= \\
& =\arctan \left[\frac{\sin \left(t+t_{1}\right)-k \cdot \cos \left(t_{1}+t_{2}\right)}{\cos \left(t+t_{1}\right)+k \cdot \sin \left(t_{1}+t_{2}\right)}\right]
\end{aligned}
$$


The slope of the circle expressed in radian $\left(\mu_{2}(t)\right)$ is constant, due to the fact that the coordinate system is fixed to the tool. Therefore, the angular deviation $\varphi(t, k)$ must be the difference between a constant and Eq. (21). If the constants are $t_{1}+t_{2}-\pi / 2=0$, then the angular deviation is certainly in the range of $-\pi / 2 \leq \varphi(t, k) \leq \pi / 2$. Thus, one of the constants, i.e. $t_{1}$ or $t_{2}$, can be chosen arbitrarily, and consequently no correction is necessary. The derivative function of the angular deviation is expressed with Eq. (22).

$$
\begin{aligned}
& \partial \varphi(t, k) / \partial t=\partial\left(\mu_{2}(t)-\mu_{1}(t, k)\right) / \partial t= \\
& =\left(1-k \cdot \sin \left(t-t_{2}\right)\right) /\left(-1+2 k \cdot \sin \left(t-t_{2}\right)-k^{2}\right)
\end{aligned}
$$

It can be seen that Eqs. (11) and Eq. (22) are identical. It can also be proved that the maximal or minimal values of the angular deviation would result in Eqs. (15a) and (15b).

\subsection{Deduction Based on Speed Vectors}

Another approach can be established where the speed vectors are examined. In this case, the feed direction and the angular position of the tool are arbitrary. Furthermore, the use of the arcus tangent function can also be avoided, so correction is unnecessary. The speed of tool tip, $\left(\overline{v_{r}}\right)$ is determined (see Fig. 8) by the linear motion resulting from the feed $\left(\overline{v_{f}}\right)$ and the circumferential speed resulting from the rotation $\left(\overline{v_{r o t}}\right)$ in Eq. (23):

$\overline{v_{r}}=\overline{v_{f}}+\overline{v_{r o t}}$

The circumferential speed can be expressed by Eq. (24), where a constant rotational speed $(S[\mathrm{rpm}])$ is assumed:

$\left|\overline{v_{r o t}}\right|=v_{r o t}=2 \pi \cdot S \cdot r=\Omega \cdot r$

The speed of feed is expressed by Eq. (25):

$\left|\overline{v_{f}}\right|=v_{f}=S \cdot f=\Omega \cdot(r / k)$

The speed vectors of feed and rotation enclose the following angle, Eq. (26):

$\beta=\pi+\left(t_{2}-t-\pi / 2\right)=t_{2}-t+\pi / 2$ (27):

Based on Fig. 8, the law of cosines can be used in Eq.

$v_{r}^{2}=v_{r o t}^{2}+v_{f}^{2}-2 \cdot v_{r o t} \cdot v_{f} \cdot \cos (\beta)$

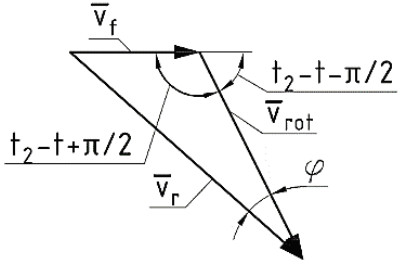

Figure 8 Depiction of the speed vectors

As shown in Fig. 8, the angular deviation $\varphi(t, k)$ is the angle enclosed by the speed vector of a point on the cutting edge and circumferential speed. The law of cosines can again be used, which is solved to $\varphi(t, k)$ in Eq. (28):

$\varphi(t, k)= \pm \arccos \left(\left(v_{f}^{2}-v_{r o t}^{2}-v_{r}^{2}\right) /\left(-2 \cdot v_{f} \cdot v_{r}\right)\right)$

Substituting Eq. (27) into Eq. (28) the following equation can be formulated as Eq. (29):

$\varphi(t, k)= \pm \arccos \left(\frac{v_{r o t}+v_{f} \cdot \cos (\beta)}{\sqrt{v_{f}^{2}+v_{r o t}^{2}+2 \cdot v_{f} \cdot v_{r o t} \cdot \cos (\beta)}}\right)$

The substitution of Eqs. (24), (25), and (26) into Eq. (29) yields Eq. (30):

$\varphi(t, k)= \pm \arccos \left(\frac{\left(k+\sin \left(t_{2}-t\right)\right)}{\sqrt{1+k^{2}+2 k \cdot \sin \left(t_{2}-t\right)}}\right)$

After simplification of the derivative function of Eq. (30), one gets the following equation, Eq. (31):

$\partial \varphi(t, k) / \partial t= \pm \frac{-1-k \cdot \sin \left(t_{2}-t\right)}{1+2 k \cdot \sin \left(t_{2}-t\right)+k^{2}}$

When choosing the minus sign in Eq. (31), Eq. (31) and Eq. (22) are identical. Therefore, if the speed vectors are considered, the same results are obtained as in Sections 1.1 and 1.2 .

\section{CIRCULAR FEED}

The investigation can also be extended to the case when the path of the centrepoint of the tool is a circle with radius $P$ (Greek rho). The radius of the circle of the tool path can be decomposed into two parts, Eq. in (32) (Fig. 9):

$P=\rho \pm R=\rho+\sigma \cdot R$

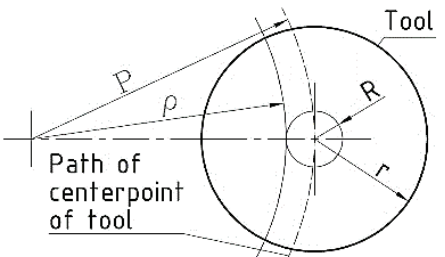

Figure 9 Decomposition of the radius of the toolpath 
The movement of the tool can be described as follows: a circle with radius $R$ rolls on the circle with radius $\rho$. In Eq. (32), $\sigma=-1$ means that this rolling movement follows the inner side of the circle, while $\sigma=+1$ means that this rolling movement follows the outer side of the circle. In the case of circular pocket milling, $\sigma=-1$ means downmilling and $\sigma=+1$ means up-milling. In the case of spigot milling, $\sigma=+1$ means down-milling and $\sigma=-1$ means up-milling. The angular velocity of circle with radius $R$ is $\theta(t)$ and the circle with radius $\rho$ is $\psi(t)$. Because of the rolling movement, the two angular velocities are equal:

$$
R \cdot \theta(t)=\rho \cdot \psi(t)
$$

The ratio of the two angular velocities can be expressed with the help of $\kappa$ in Eq. (34):

$$
\kappa=\rho / R=\theta(t) / \psi(t)
$$

The path of the centrepoint of the tool can be expressed in function of $(r, k, \kappa)$ in Eq. (35), by substituting Eqs. (4) and (34) into Eq. (32):

$$
P=(r / k) \cdot(1+\sigma \cdot \kappa)
$$

The real angular velocity of the tool $\tau(t)$ can be broken, on the one hand, into $\psi(t)$ resulting from the feed, and, on the other hand, into $\theta(t)$ resulting from the rolling circle, as it is expressed by Eq. (36a). With the help of $\kappa$, the real angular velocity of the tool $\tau(t)$ can be expressed using $\theta(t)$ or $\psi(t)$, as expressed by Eq. (36b) or Eq. (36c), respectively.

$$
\begin{aligned}
& \tau(t)=\sigma \cdot \psi(t)+\theta(t) \\
& \tau(t)=\theta(t) \cdot((\kappa+\sigma) / \kappa) \\
& \tau(t)=\psi(t) \cdot(\kappa+\sigma)
\end{aligned}
$$

The two angular velocities, $\theta(t)$ and $\psi(t)$ can be expressed using $\tau(t)$ with the help of Eq. (36b) and (36c), respectively Eqs. (37a) and (37b):

$$
\begin{aligned}
& \theta(t)=\tau(t) \cdot \kappa /(\kappa+\sigma) \\
& \psi(t)=\tau(t) /(\kappa+\sigma)
\end{aligned}
$$

The circumferential speed of the centrepoint of the tool is expressed by Eq. (38), using Eq. (34), (35) and (36c):

$$
\begin{aligned}
& v_{f}=P \cdot \psi(t)=P \cdot \theta(t) / \kappa= \\
& =(r / k) \cdot(1+\sigma \cdot \kappa) \cdot \tau(t) /(\kappa+\sigma)=\sigma \cdot(r / k) \cdot \tau(t)
\end{aligned}
$$

Due to the fact that $\tau(t)$ is the actual angular velocity of the tool, the feed per rotation, $f$, can be expressed as follows in Eq. (39):

$$
f=\sigma \cdot(r / k) \cdot 2 \pi
$$

The path of a point on the cutting edge with radius $r$ is an epicycloid or a hypocycloid, and can be described by functions $X(t)$, and $Y(t)$ with the help of the conversion of Eqs. (6) and (7), respectively, as follows in Eqs. (40) and (41):

$$
\begin{aligned}
& X(t)=P \cdot \cos (\sigma \cdot \psi(t))+r \cdot \cos (\tau(t)) \\
& Y(t)=P \cdot \sin (\sigma \cdot \psi(t))+r \cdot \sin (\tau(t))
\end{aligned}
$$

The substitution of Eqs. (34) and (35) into Eqs. (40) and (41), respectively, yields the slope expressed in radian, in which case the variable is $\tau(t)$, as described in the following equation, Eq. (42):

$$
\begin{aligned}
& \mu_{1}(t, k, \kappa)=\arctan \left(\frac{Y^{\prime}(t)}{X^{\prime}(t)}\right)= \\
& =-\arctan \left[\frac{\cos (\tau(t) \cdot \sigma /(\kappa+\sigma)) \cdot \sigma+\cos (\tau(t)) \cdot k}{\sin (\tau(t) \cdot \sigma /(\kappa+\sigma)) \cdot \sigma+\sin (\tau(t)) \cdot k}\right]
\end{aligned}
$$

The slope in radian can be expressed by Eq. (43) in function of $\theta(t)$ with the help of substituting Eq. (36b) into Eq. (42):

$$
\begin{aligned}
& \mu_{1}(t, k, \kappa)=\arctan \left(\frac{Y^{\prime}(t)}{X^{\prime}(t)}\right)= \\
& =-\arctan \left[\frac{\cos (\theta(t) \cdot \sigma / \kappa) \cdot \sigma+\cos (\theta(t) \cdot(\kappa+\sigma) / \kappa) \cdot k}{\sin (\theta(t) \cdot \sigma / \kappa) \cdot \sigma+\sin (\theta(t) \cdot(\kappa+\sigma) / \kappa) \cdot k}\right]
\end{aligned}
$$

The angular rotation in function of $t$ is $\tau(t)$ in Eq. (42) and that is $\theta(t)$ in Eq. (43). In both equations the constants are $k, \kappa, \sigma$. The curve described by Eqs. (40) and (41) is directed, therefore, its slope expressed in radian has to be in the range of $-\pi \leq \mu_{1} \leq \pi$. Due to the arcus tangent function, the slope expressed in radian is in the range of $-\pi / 2 \leq \mu_{1} \leq \pi / 2$, thus correction is necessary. The sign and zero of $X^{\prime}(t)$ and $Y^{\prime}(t)$ should be determined for the correction. However, finding the zeros of the established equations, i.e. $X^{\prime}(t)=0$ and $Y^{\prime}(t)=0$, cannot be solved analytically, thus Eq. (8) should be used.

The equation and slope of the circle expressed in radian can be formulated with the help of $\tau(t)$ and $\theta(t)$. Angular deviation $\varphi(t, k, \kappa)$ can be expressed using the difference between the slopes of the circle and that of the cycloid curve expressed in radian as follows:

$$
\begin{aligned}
& \varphi(t, k, \kappa)=\mu_{2}(t, \kappa)+\operatorname{corr}_{2}(t, k, \kappa)- \\
& -\mu_{1}(t, k, \kappa)-\operatorname{corr}_{1}(t, k, \kappa)
\end{aligned}
$$

In Eq. (44), the variable is $\tau(t)$ or $\theta(t)$ and the constants are $k, \kappa, \sigma$. It should be noted that the angular deviation is independent of $\kappa$, because its derivative function is zero, i.e. $\partial \varphi(t, k, \kappa) / \partial \kappa=0$. The maxima and minima of the angular deviation can be determined with the 
help of the derivative function of Eq. (44). After simplification, the following equation can be established for variables $\theta(t)$ and $\tau(t)$, respectively in Eqs. (45) and (46):

$$
\begin{aligned}
& \partial \varphi(t, k, \kappa) / \partial t=\frac{-1-\sigma \cdot k \cdot \cos (\theta(t))}{1+2 k \cdot \sigma \cdot \cos (\theta(t))+k^{2}} \\
& \partial \varphi(t, k, \kappa) / \partial t=\frac{-1-\sigma \cdot k \cdot \cos (\tau(t) \cdot \kappa /(\kappa+\sigma))}{\left(1+2 k \cdot \sigma \cdot \cos (\theta(t))+k^{2}\right) \cdot(\kappa+\sigma)}
\end{aligned}
$$

After substituting Eq. (37a) into Eq. (46) and multiplying it by $(\kappa /(\kappa+\sigma))$, Eq. (45) is equal to Eq. (46). The denominators of Eqs. (45) and (46) are certainly greater than 0 in the case of $1<k$, therefore, the functions are continuous. The zeros are expected to be located where the numerators of Eqs. (45) and (46) are equal to zero. After simplification, the following, Eqs. (47) and (48), can be obtained:

$$
\begin{aligned}
& \theta(t)= \pm \arccos (-1 /(\sigma \cdot k)) \\
& \tau(t)= \pm(\arccos (-1 /(\sigma \cdot k))) \cdot(\kappa /(\kappa+\sigma))
\end{aligned}
$$

By substituting Eq. (47) into Eq. (45), and Eq. (48) into Eq. (46), as well as using $\arctan (\cot (x))=x+(2 n+1 / 2) \cdot \pi$ and $\arctan (\tan (x))=x+2 n \pi$, where $n$ is an integer, the following, Eqs. (49a) and (49b) can be concluded, respectively:

$$
\begin{aligned}
& \Phi(k, \sigma)=\pi / 2-\arccos (1 /(\sigma \cdot k))=\arcsin (1 /(\sigma \cdot k)) \\
& \Phi(k, \sigma)=-3 / 2 \pi+\arccos (1 /(\sigma \cdot k))= \\
& =-\pi-\arcsin (1 /(\sigma \cdot k))
\end{aligned}
$$

Accordingly, the maximum or minimum of the angular deviation is the function of $k$ and $\sigma$, and is independent of the radius of the toolpath $(\kappa)$. The result is the same, either angular velocity $\tau(t)$ or $\theta(t)$ is used.

Finally, the angular deviation in the case of circular feed, i.e. Eqs. (49a) and (49b), is equal to the angular deviation in the case of linear feed, i.e. Eqs. (14a) and (14b), if $\Phi(k, \sigma)=\Phi(k, \sigma)+2 n \pi$, where $n$ is an integer.

\section{APPLICATION AND EXPERIMENT DESIGN}

In many experiments and in the case of tool path generation, the exit angle has so far been calculated by way of approximation. With the help of Eq. (15ab) the error of the approximation can be determined. From Eqs. (15a) and (15b) $f$ can be expressed in the function of $r$ for an arbitrary $\Phi$.

If the tolerated difference between the theoretical and approximated angle values: $|\Phi|=1^{\circ}$, then the range of applicable feed rate for any tool radius is wide and considerably covers the usual values (Fig. 10). Thus, the approximating calculation, i.e. the substitution of cycloid curve with a circle, can be a useful method in practice, both for determining the exit angle and for toolpath generation.

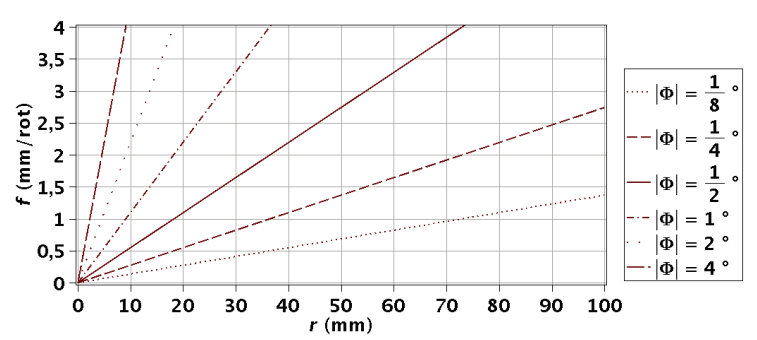

Figure 10 Permissible feed rates at different maximum angular deviation in the function of tool radius

As displayed in Fig. 2, it is not only the exit angle $\alpha$ which changes with the translation of the tool perpendicular to the workpiece contour but also the chip thickness. It seems reasonable to design an experiment where either the exit angle or the chip thickness changes. To do this, a special workpiece geometry is a possible solution, in which case the tool moves in a straight line at a constant feed while the exit angle is approximately constant but the chip thickness changes. A path of a point on the cutting edge can be approximated using a circle, as the deviation of the exit angle is known. This approximation provides a viable, analytical solution. The exit angle can be determined as the angle enclosed by the tangent lines of the curve to be identified $(y=f(x))$ and the circle that moves along a line. In the simplest case, the tangent lines are perpendicular and $\alpha=\pi / 2$, thus the following differential equation can be used:

$$
\frac{\mathrm{d} y(x)}{\mathrm{d} x}=\frac{y(x)}{\sqrt{r^{2}-y(x)^{2}}}
$$

The initial conditions are: $y(0)=r$ and $y^{\prime}(0)=\infty$. In fact, Eq. (50) does not have an explicit solution. However, the curve can be defined using the following parametric form in Eq. (51):

$$
\frac{y^{\prime}(t)}{x^{\prime}(t)}=\frac{y(t)}{\sqrt{r^{2}-y(t)^{2}}}
$$

One of the two component equations, $x(t)$ or $y(t)$, is arbitrary. In the case of $x(t)=t$, no solution exists, but in the case of $y(t)=t$, there exists a solution, Eq. (52):

$$
\begin{aligned}
& x(t)=\int \frac{\sqrt{r^{2}-t^{2}}}{t \mathrm{~d} t}= \\
& \sqrt{r^{2}-t^{2}}-r \cdot \ln \left(\frac{2 r \cdot\left(1+\sqrt{r^{2}-t^{2}}\right)}{t}\right)+C
\end{aligned}
$$

Eq. (52) is valid if $\alpha=\pi / 2$. However, the exit angle can move in a range of $0 \leq \alpha \leq \pi$, thus another solution is necessary. A circle can be described with a radius $r$ and an angular parameter $t$ (e.g. $y(t)=r \cdot \sin (t))$. The slope of the curve is equal to the sum of the slope of the circle and the exit angle at $\pi / 2$ in Eq. (53) as follows:

$$
y^{\prime}(t) / x^{\prime}(t)=\tan (t-\pi / 2+\alpha)=-\cot (t+\alpha)
$$


Substituting one of the circle equations $y(t)=r \cdot \sin (t)$ into Eq. (53), unknown $x(t)$ can be expressed analytically as follows in Eq. (54):

$$
\begin{aligned}
& x(t)=\int \frac{-r \cdot \cos (t)}{\cot (t+\alpha)} \mathrm{d} t= \\
& =r \cdot\left[1+\cos (t)+2 \sin (\alpha) \tanh ^{-1}\left(\frac{\cos (\alpha)-\cos (t-\alpha)}{\sin (t)}\right)\right]
\end{aligned}
$$

By substituting $\alpha=\pi / 2$ into Eq. (54), the following simple form can be obtained in Eq. (55):

$$
\begin{aligned}
& x(t)=r \cdot\left(\cos (t)+\ln \left(\frac{1-\cos (t)}{\sin (t)}\right)\right) \\
& \cdots \alpha=\frac{1}{12} \pi \cdot \alpha=\frac{1}{6} \pi-\cdots \alpha=\frac{1}{4} \pi-\alpha=\frac{1}{3} \pi-\alpha=\frac{5}{12} \pi-\alpha=\frac{1}{2} \pi \\
& -\alpha=\frac{7}{12} \pi \cdots \alpha=\frac{2}{3} \pi \cdot \alpha=\frac{3}{4} \pi \cdot \alpha=\frac{5}{6} \pi-\alpha=\frac{11}{12} \pi \\
& r=1
\end{aligned}
$$

Figure 11 The graphs of Eqs. (54) and (55)

Fig. 11 shows some detailed curves that belong to different exit angles. In Fig. 12, an experimental workpiece geometry is shown, where the tool moves along a line and the exit angle is in the range of $\pi / 2-\Phi(f) \leq \alpha \leq \pi / 2+\Phi(f)$.

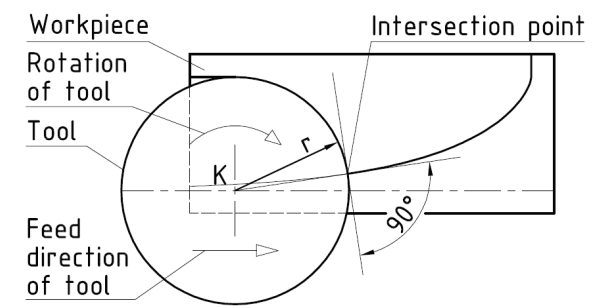

Figure 12 Experimental workpiece geometry for the examination of $\alpha=\pi / 2$

\section{CONCLUSIONS}

The calculation of the exit angle is simplified by applying a special calculation method that relies on the approximation of the path of a point on the cutting edge with a circle. The maximal error of this approximation can be accurately determined with the help of the new expressions, Eqs. (14a) and (14b), and it can likewise be reliably estimated with the help of the new expressions, Eqs. (16a) and (16b). The error directly increases with the rise in the feed per rotation. These equations are confirmed by two methods: by using a coordinate system fixed to the tool and with the help of the speed vectors. Both methods produce the same result.

Moreover, the applicability of the equations can be extended to the cases when circular feed is used. Furthermore, as has been shown, the error is independent of the radius of the toolpath.
In addition, the range within which the error of the approximately calculated exit angle falls can be accurately determined with Eq. (56).

$$
\left|\alpha_{r}-\alpha_{t}\right|=|\varphi(f)| \leq \Phi(f)
$$

The width of this range only depends on the feed rate. This range can be used in the case of any toolpath that is constructed or approximated using lines and circles.

The proposed deduction ensures that the approximated calculation of exit angle is useful in a wide range of feed rates and the deviation is not significant in the case of usual feed rates. The usage of circle to estimate the exit angle is suitably accurate for practical use. This approximation method can also be efficiently used for toolpath generation when the exit angle is considered. It was demonstrated by experiments [21].

With the help of the approximated calculation of the exit angle, special workpiece geometry has been designed: in this case the tool moves along a line while the exit angle is almost constant and the effects of the change of chip thickness on burr formation can be examined.

\section{Acknowledgements}

Work for this paper was supported by the European Commission through the $\mathrm{H} 2020$ project EPIC under grant No. 739592 and by the Higher Education Excellence Program of Ministry of Human Capacities in the frame of Artificial Intelligence research area of the Budapest University of Technology and Economics (BME FIKPMI).

\section{REFERENCES}

[1] Amrendra, K. Y., Mohan, K., Vivek, B., Nirmal, K. S., \& Ramesh, K. S. (2017). FE modeling of burr size in highspeed micro-milling of Ti6A14V. Precision Engineering, 49, 287-292. https://doi.org/10.1016/j.precisioneng.2017.02.017

[2] Arrazola, P. J., Özel, T., Umbrello, D., Davies, M., \& Jawahir, I. S. (2013). Recent advances in modelling of metal machining processes. CIRP Annals - Manufacturing Technology, 62, 695-718. https://doi.org/10.1016/j.cirp.2013.05.006

[3] Aurich, J. C., Dornfeld, D. A., Arrazola, P. J., Franke, V., Leitz, L., \& Min, S. (2009). Burrs-Analysis, control and removal. CIRP Annals - Manufacturing Technology, 58, 519-542. https://doi.org/10.1016/j.cirp.2009.09.004

[4] Chern, G. L. (2006). Study on Mechanisms of Burr Formation and Edge Breakout near the Exit of Orthogonal Cutting. Journal of Materials Processing Technology, 176, 152-157. https://doi.org/10.1016/j.jmatprotec.2006.03.127

[5] Chern, G. L. (2006). Experimental observation and analysis of burr formation mechanisms in face milling of aluminum alloys. International Journal of Machine Tools \& Manufacture, 46, 1517-1525. https://doi.org/10.1016/j.jimachtools.2005.09.006

[6] Chu, C. H. \& Dornfeld, D. A. (2000). Tool path planning for avoiding exit burrs. Journal of Manufacturing Processes, 2(2), 116-123. https://doi.org/10.1016/S1526-6125(00)70019-3

[7] Gillespie, L. K. \& Blotter, P. T. (1976). The Formation and Properties of Machining Burrs. Transactions of ASME Journal of Engineers for Industry, 98, 66-74. https://doi.org/10.1115/1.3438875 
[8] Hashimura, M., Hassamontr, J., \& Dornfeld, D. A. (1999). Effect of in-plane exit angle and rake angles on burr height and thickness in face milling operation. Journal of Manufacturing Science and Engineering, 121, 13-19. https://doi.org/10.1115/1.2830566

[9] Hassamontr, J., Blondaz, L., \& Dornfeld, D. A. (1998) Avoiding Exit Burrs in CNC End Milling by an Adapted Tool-Path. Proceedings of the ASME IMECE, Anaheim, 497-501.

[10] Jones, S., \& Furness, R. (1997). An Experimental Study of Burr Formation for Face Milling 356 Aluminium. Transactions of NAMRI/SME, 25, 183-188.

[11] Kiswanto, G., Zariatin, D. L., \& Ko, T. J. (2014). The effect of spindle speed, feed-rate and machining time to the surface roughness and burr formation of Aluminium Alloy 1100 in micro-milling operation. Journal of Manufacturing Processes, 16, 435-450. https://doi.org/10.1016/j.jmapro.2014.05.003

[12] Kou, Z., Wan, Y., Cai, Y., Liang, X., \& Liu, Z. (2015). Burr Controlling in Micro Milling with Supporting Material Method. Procedia Manufacturing, 1, 501-511. https://doi.org/10.1016/j.promfg.2015.09.015

[13] Kumar, S. (2001). Basic Model for Burr Prediction System. Master's Report. University of California, Berkeley, CA.

[14] Lekkala, R., Bajpai, V., Singh, K. R., \& Joshi, S. S. (2011). Characterization and modelling of burr formation in microend milling. Precision Engineering, 35, 625-637. https://doi.org/10.1016/j.precisioneng.2011.04.007

[15] Martellotti, M. E. (1941). An Analysis of the Milling Process. Trans. ASME, 63, 677-700.

[16] Martellotti, M. E. (1945). An Analysis of the Milling Process, Part 2. Down milling. Trans. ASME, 67, 233-251.

[17] Narayanaswami, R. \& Dornfeld, D. A. (1997). Burr Minimization in Face Milling: A Geometric Approach. ASME Journal of Manufacturing Science and Engineering, 119, 170-177. https://doi.org/10.1115/1.2831092

[18] Niknam, S. A. \& Songmene, V. (2013). Experimental investigation and modelling of milling burrs. Proceedings of the ASME 2013 International Manufacturing Science and Engineering Conference MSEC2013, Madison, Wisconsin, USA. https://doi.org/10.1115/MSEC2013-1176

[19] Niknam, S. A. \& Songmene, V. (2014). Analysis of friction and burr formation in slot milling. Procedia CIRP, 17, 755759. https://doi.org/10.1016/j.procir.2014.02.032

[20] Olvera, O. \& Barrow, G. (1996). An Experimental Study of Burr Formation in Square Shoulder Face Milling. International Journal of Machine Tools and Manufacture, 36(9), 1005-1020. https://doi.org/10.1016/0890-6955(96)00014-4

[21] Póka, Gy., Mátyási, Gy., \& Németh, I. (2016). Burr minimisation in face milling with optimised tool path. Procedia CIRP, 57, 653-657. https://doi.org/10.1016/j.procir.2016.11.113

[22] Preś, P., Skoczyński, W., \& Jaśkiewicz, K. (2014). Research and modelingworkpiece edge formation process during orthogonal cutting. Archives of Civil and Mechanical Engineering, 14, 622-635. https://doi.org/10.1016/j.acme.2014.01.003

[23] Rangarajan, A. (2001). Optimization of the Face Milling Process: Tool Path and Tool Design Issues. Part 1. Algorithm and Tool Path Design LMA. Research Reports, University of California, Berkeley, CA, 1-8.

[24] Silva, L. C., Mota, P. R., \& Silva, M. B., Ezugwu, E. O., Machado, A. (2015). Study of burr behavior in face milling of PH 13-8 Mo stainless steel. CIRP Journal of Manufacturing Science and Technology, 8, 34-42. https://doi.org/10.1016/j.cirpj.2014.10.003

[25] Silva, L. C., Mota, P. R., Silva, M. B., Ezugwu, E. O., \& Machado, A. (2015). Study of burr height in face milling of PH 13-8 Mo stainless steel -Transition from primary to secondary burr and benefits of deburring between passes. CIRP Journal of Manufacturing Science and Technology, 10, 61-67. https://doi.org/10.1016/j.cirpj.2015.05.002

[26] Spiewak, S. (1995). An Improved Model of the Chip Thickness in Milling. Ann. CIRP, 44(1), 39-42. https://doi.org/10.1016/S0007-8506(07)62271-9

[27] Wang, G. C. \& Zhang, C. Y. (2004). Mechanism of Burr Formation in Milling. Key Engineering Materials, 259-260 : 278-281 https://doi.org/10.4028/www.scientific.net/KEM.259-260.278

[28] Wojciechowski, S., Chwalczuka, T., Twardowskia, P., \& Krolczyk, G. M. (2015). Modeling of cutter displacements during ball end milling of inclined surfaces. Archives of Civil and Mechanical Engineering, 15, 798-805. https://doi.org/10.1016/j.acme.2015.06.008

[29] Wu, X., Li, L., \& He, N. (2017). Investigation on the burr formation mechanism in micro cutting. Precision Engineering, 47, 191-196. https://doi.org/10.1016/j.precisioneng.2016.08.004

\section{Nomenclature}

$\alpha$ the exit angle

$\alpha_{r}$ the real value of exit angle

$\alpha_{t}$ the theoretical value of exit angle

$f$ feed per revolution

$r$ tool radius

$R$ radius of the rolling circle

$k \quad$ ratio of the rolling circle and tool radius

$P \quad$ radius of path of tool centrepoint

$\rho$ radius of stationary circle

$\kappa \quad$ ratio of $\rho$ and $R$

$t$ time parameter in the equation, it refers to the rotation of the tool

$t_{0}, t_{1}, t_{2}, t_{3}$ constants of the equation of the cycloid curve $t_{m}$ examined time moment

$\mu_{1}$ slope of cycloid curve in radian

$\mu_{2}$ slope of circle in radian

$\varphi \quad$ angular deviation between $\mu_{1}$ and $\mu_{2}$

$\Phi$ maximum or minimum of $\varphi$

\section{Contact information:}

\section{György PÓKA}

Budapest University of Technology and Economics, Department of Manufacturing Science and Engineering, H-1111 Budapest, Müegyetem rkp. 3, Hungary poka@manuf.bme.hu

\section{István NÉMETH, PhD}

Budapest University of Technology and Economics, Department of Manufacturing Science and Engineering, H-1111 Budapest, Müegyetem rkp. 3, Hungary inemeth@manuf.bme.hu 